\title{
Intakes of plant foods, fibre and fat and risk of breast cancer - a prospective study in the Malmö Diet and Cancer cohort
}

\author{
I Mattisson*,', E Wirfält', U Johansson', B Gullberg'², H Olsson ${ }^{3}$ and G Berglund' \\ 'Department of Medicine, Surgery and Orthopaedics, Lund University, Malmö University Hospital, SE-205 02 Malmö, Sweden; '²Department of \\ Community Medicine, Lund University, Malmö University Hospital, Entrance 59, SE-205 02 Malmö, Sweden; ${ }^{3}$ Department of Oncology, Lund University, \\ SE-22I 85 Lund, Sweden
}

The objective of this study was to investigate prospectively the associations between intakes of plant foods, fibre and relative fat and risk of breast cancer in a subsample of I 726 postmenopausal women in the Malmö Diet and Cancer cohort. Data were obtained by an interview-based diet history method, a structured questionnaire, anthropometrical measurements and national and regional cancer registries. During 89602 person-years of follow-up, 342 incident cases were documented. Cox regression analysis examined breast cancer risks adjusted for potential confounders. High fibre intakes were associated with a lower risk of postmenopausal breast cancer, incidence rate ratio $=0.58,95 \% \mathrm{Cl}$ : $0.40,0.84$, for the highest quintile of fibre intake compared to the lowest quintile. The combination high fibre-low fat had the lowest risk when examining the effect in each cell of cross-classified tertiles of fibre and fat intakes. An interaction $(P=0.049)$ was found between fibre- and fat-tertiles. There was no significant association between breast cancer risk and intakes of any of the plant food subgroups. These findings support the hypothesis that a dietary pattern characterised by high fibre and low fat intakes is associated with a lower risk of postmenopausal breast cancer. British Journal of Cancer (2004) 90, 122 - 127. doi:I0.1038/sj.bjc.660 I 5 I 6 www.bjcancer.com (C) 2004 Cancer Research UK

Keywords: fat; fibre; plant foods; postmenopausal breast cancer; prospective study

Breast cancer is the most common cancer in women worldwide, but incidence rates vary greatly between countries (World Cancer Research Fund, 1997). Several biological processes associated with diet might be relevant to breast cancer aetiology. Much of the previous research on breast cancer and diet has focused on the total fat intake and disease risk, but results from different studies are conflicting (Lee and Lin, 2000; Willett, 2001). Plant foods have attracted less interest, although they are sources of several compounds that might be of importance in breast cancer development. These compounds include vitamins (e.g., ascorbic acid, folic acid, vitamin $\mathrm{E}$ and $\beta$-carotene), fibre and various phytochemicals (flavonoids, phyto-oestrogens, indols, carotenoids, etc.). Phyto-oestrogens especially have the potential to influence breast cancer risk. They are similar in structure to oestrogen and may compete with endogenous oestrogen for the oestrogen receptors (Cassidy et al, 2000).

High fat consumers in the Malmö Diet and Cancer (MDC) cohort have substantially lower intakes of fruit, vegetables and whole grain bread compared to low fat consumers and intakes of antioxidants and fibre are inversely associated with the relative fat intake (Mattisson et al, 2003a). Thus, it is of interest to examine if the association between plant food intake and breast cancer risk is influenced by fat intake. The aims of this study are to examine (1) if plant foods and fibre intakes are associated with breast cancer

\footnotetext{
* Correspondence: Dr I Mattisson, Malmö Diet and Cancer Study, UMAS Entrance 59, SE-205 02 Malmö, Sweden;

E-mail: irene.mattisson@smi.mas.lu.se

Received 28 July 2003; revised 10 October 2003; accepted 15 October 2003
}

risk, and (2) if these associations are influenced by fat intake. In addition, the combined effect of fat and fibre intakes on breast cancer incidence was examined by cross-classifying energyadjusted tertiles of fat and fibre.

\section{MATERIAL AND METHODS}

\section{Malmö Diet and Cancer}

The MDC study is a prospective cohort study in Malmö, a city in the south of Sweden with approximately 250000 inhabitants. The MDC source population was, in 1991, defined as all persons living in the City of Malmö, born between 1926 and 1945. However, in May 1995, the cohort was extended to also include all women born between 1923 and 1925 and 1946 and 1950, and all men born between 1923 and 1925. With this extension, 74138 persons constitute the source population. Both personal invitation letters and passive recruitment strategies (e.g., advertisement in newspapers and on buses) directed towards the community were used to recruit study participants. During the first 2 years, approximately $50 \%$ of the subjects were recruited by passive recruitment. However, during 1994-1996, less than 5\% were passively recruited. Inadequate Swedish language skills and mental incapacity were the only exclusion criteria. When the baseline examinations closed in October 1996, 28098 participants had completed all parts. More details of recruitment and the cohort are described elsewhere (Manjer et al, 2002). The ethical committee at Lund University has approved the MDC (LU 51-90).

The participants visited the MDC screening centre twice. During the first visit, groups of 6-8 participants were informed about the 
study, instructed how to register meals in the menu-book and how to fill out the diet questionnaire and the extensive questionnaire covering socio-economic factors and lifestyle. Project nurses took blood samples, blood pressure and anthropometric measurements. Participants completed all questionnaires at home. The dietary interview was conducted and the socio-economic questionnaire checked at the second visit, approximately 10 days after the first.

\section{Study population}

In total, 11726 postmenopausal women were included in this subsample from the MDCS cohort. This study used an age-based definition of menopausal status (Morabia and Flandre, 1992). Eligible participants for this analysis were women who were 50 years or older at baseline examination. All prevalent cancer cases, except cervix cancer in situ and squamous cell carcinoma of the skin, were excluded. Women born between 1923 and 1925 and 1946 have shorter follow-up, due to the MDC study design.

\section{Case definition and ascertainment}

The National Swedish Cancer Registry provided data until December 1999; additional information until end of follow-up (31 December 2001) was obtained from the Southern Swedish Regional Tumour Registry. Cases were women diagnosed during follow-up with invasive breast cancer or breast cancer in situ. Information on vital status was obtained from the National Tax Board that provides up-to-date information on vital status for all Swedish residents. Cases contributed person-time from date of enrolment until time of diagnosis. Noncases contributed persontime from the date of enrolment until death (470 women), migrating from Sweden (57 women) or end of follow-up (31 December 2001), whichever was the first. In total, 312 invasive and 30 in situ incident breast cancer cases were documented during 89602 years of follow-up.

\section{Dietary data}

The MDC method is an interview-based, modified diet history method. Briefly, it combines a 7-day menu book for recording of cooked meals, beverages, drugs, natural remedies and nutrient supplements and a diet questionnaire for assessment of meal pattern, consumption frequencies and portion sizes of regularly eaten foods. The mean daily intakes of food were calculated and converted to energy and nutrient intakes using PCKost2-93 from the National Food Administration in Uppsala, Sweden. The MDC method is described in detail elsewhere (Mattisson et al, 2001; Wirfält et al, 2002b). The relative validity of the MDC method was evaluated in a sample of Malmö residents, 105 women and 101 men, 50-69 years old, using 3 days of weighed records every other month during a year, as a reference method. The energy-adjusted Pearson correlation coefficients, between the reference method and the MDC method administered after the 12month reference period, were 0.69 for both fibre and fat intake, (Riboli et al, 1997), 0.53 for vegetables, 0.77 for fruit, 0.51 for potatoes, 0.58 for bread and 0.24 for rice and pasta (Elmståhl et al, 1996).

\section{Variables}

This study examined gram amounts of fibre and of the following 16 plant food-group variables: total fruit (including berries), fruit juice, vegetables, total fruit and vegetables, total fruit, vegetables and fruit juice, potatoes boiled, potatoes fried and deep fried, highfibre bread, low-fibre bread, total bread, high-fibre cereals, lowfibre cereals, buns and cookies, nuts, snacks and rice and pasta.
Total fat, n-6 polyunsaturated fatty acids (PUFAs), wine and fermented milk were also examined as potential confounders because these variables have been associated with breast cancer risk in previous studies in the MDC cohort (Wirfält et al, 2002a; Mattisson et al, 2003b; Wirfält et al, 2003).

Energy-adjusted variables of total fat, n-6 PUFAs, fibre, total fruit and vegetables and fermented milk variables were defined as the residuals obtained when regressing the specific nutrient or food group on total energy intake (EI). Five exposure categories were created based on the quintile ranking of participants on residuals. In addition, for fat and fibre, a threecategory variable was defined, based on the tertile ranking of participants on residuals. Information on wine consumption $\left(\mathrm{clday}^{-1}\right)$ was converted into a four-category variable based on the ranking of absolute intakes: wine-abstainers (zero consumption in menu book and no wine consumption during the last year indicated in the questionnaire); low consumers (wine intakes below or equal to the median intake); medium consumers (wine intakes above median intake but below or equal to the 97.5th percentile; high consumers (wine intakes above the 97.5th percentile).

In September 1994, the dietary data processing procedure was slightly altered (Wirfält et al, 2002b). Method version (indicating data collection before or after 1 September 1994), diet interviewer and season of diet interview were used as covariates to control for undue variation in the dietary data collection over time.

Age at baseline was obtained from the 10-digit person identification number.

Information on reproduction, socio-economic and life style factors was collected by a structured multiple-choice questionnaire.

Past change of dietary habits was based on the question 'have you substantially changed your dietary habits because of illness or another reason?'

Age at menarche was used as a continuous variable. Age at the birth of the first child was divided into four categories ( $\leqslant 24$ years, $24-30$ years, $>30$ years and 'no children'); missing information (2.0\%) was recoded to 'no children'.

Current use of hormone therapy was based on the question 'which medicines do you use on a regular basis?' in combination with the 7-day recording of drug use in the menu book, and used as a dichotomous (yes/no) variable (Merlo et al, 2000).

Participants were divided into four categories according to their highest level of education: $\leqslant 8$ years, $9-10$ years, $11-12$ years and college education/university degree.

Participants indicated the number of minutes per week, separately for the four seasons, for 17 different physical activities (Haftenberger et al, 2002). The question was adapted from the Minnesota Leisure Time Physical Activity Questionnaire (Taylor et al, 1978; Richardsson et al, 1994). The number of minutes of each activity was multiplied with an activity coefficient and an overall leisure time physical activity score was created. The score was divided into quintiles and further categorised as low (quintile 1), moderate (quintile 2-4) or high (quintile 5).

Standing height was measured with a fixed stadiometer calibrated in centimetres. Weight was measured to the nearest $0.1 \mathrm{~kg}$ using a balance-beam scale with subjects wearing light clothing and no shoes. Waist was measured midway between the lowest rib margin and iliac crest. Owing to the high correlation $(r=0.86)$ between BMI and waist circumference, we decided to use only one obesity measure as a covariate. Waist was chosen because central obesity rather than general obesity has been suggested to predispose an individual to the development of breast cancer (Stoll, 1996).

To evaluate reported EIs, the ratio between reported total EI and basal metabolic rate (BMR) was calculated. Basal metabolic rate was estimated using the equation recommended by WHO; based on age, sex, weight and height (FAO/WHO/UNU, 1985). 


\section{Statistical methods}

SPSS statistical computer package (version 10.0; SPSS Inc., Chicago, IL, USA) was used for the statistical analyses. Crude median and interquartile intakes of food groups and fibre were calculated for cases and noncases. All dietary variables were logtransformed to reduce skewness. Before transformation, a very small amount $(=0.01)$ was added to food intake variables in order to handle zero consumption. $T$-test examined differences in the mean food and fibre intakes between cases and noncases.

The multivariate associations between food intake and breast cancer risk were examined with Cox regression models. Firstly, the influence of each of the 16 plant food-groups and dietary fibre on breast cancer risk was examined as continuous variables, while adjusting for diet interviewer, season of diet interview, method version, age at baseline, past food habit change and total energy (basic model). Food groups were further examined, in 'full models' also including established and potential risk factors (i.e., height, waist, current hormone use, age at the birth of the first child, age at menarche, leisure time physical activity and educational level). Secondly, the risk across quintiles of total fruits and vegetables and dietary fibre was estimated with both the 'basic' and the 'full' models. The 'full models' were extended to include adjustment for energy-adjusted quintiles of total fat, n-6 PUFAs, fermented milk or wine intake categories since these have been found to influence breast cancer risk in the MDC cohort. The models were also repeated while excluding women less than 51 years at baseline ( $n=719$, including 27 cases); women diagnosed with incident breast cancer within 1 year from baseline examination (31 cases) or in situ cancer cases (30 cases).

Finally, energy-adjusted tertiles of fat and fibre intakes were cross-classified, resulting in a nine-category grid. Cox regression estimated the breast cancer risk across all nine categories, with the combination high fibre-low fat as the reference category, since this category was believed to represent the lowest breast cancer risk (World Cancer Research Fund/American Institute for Cancer Research, 1997). This model was also extended to include adjustment for n-6 PUFA quintiles, wine intake categories and fermented milk quintiles. Further, we tested for interaction between tertiles of fibre and fat.

\section{RESULTS}

Table 1 shows the median intakes of the different plant foodgroups and fibre in cases and noncases. There were no significant differences in the mean intakes of food groups and fibre between cases and noncases (data not shown). The baseline characteristics of cases and noncases are described in detail elsewhere (Mattisson et al, 2003b). In short, cases were younger at baseline (a consequence of the MDC study design and the shorter follow-up among many older women), taller, more often current users of hormone therapy and had more often medium-high physical activity.

In the multivariate analysis, there were no significant associations between food group intakes (continuous) and breast cancer risk (data not shown). Fibre intake (continuous) was significantly associated with decreased risk, both in the 'basic model' $(P=0.044)$ and in the 'full model' $(P=0.012)$.

We also examined the associations between energy-adjusted quintiles of fibre and of fruit and vegetables and breast cancer risk. The highest quintile of fibre intake was associated with a lower breast cancer risk both in the basic and full models (Table 2); however, the trend across quintiles was not significant. Adjusting for wine, n-6 PUFAs or fermented milk did not influence the association between fibre and breast cancer risk (data not shown). Adjusting for total fat attenuated the association but the lower risk in the fifth fibre quintile remained significant (Table 2). None of
Table I Intakes of plant foods and fibre at baseline examination in cases and noncases in the Malmö Diet and Cancer cohort

\begin{tabular}{|c|c|c|c|c|}
\hline & \multicolumn{2}{|c|}{ Cases $(n=342)$} & \multicolumn{2}{|c|}{ Noncases $(n=$ I I 384) } \\
\hline & $\begin{array}{l}\text { Median } \\
\left(\text { g day }^{-1}\right)\end{array}$ & $\begin{array}{l}\text { IQ } \\
\text { range }\end{array}$ & $\begin{array}{l}\text { Median } \\
\left(\text { g day }^{-1}\right)\end{array}$ & $\begin{array}{l}\mathrm{IQ} \\
\text { range }\end{array}$ \\
\hline Total fruits and berries & 197 & 148 & 196 & 154 \\
\hline Total fruit juices & 0.78 & 143 & 0.64 & 107 \\
\hline Total vegetables & 154 & || $\mid$ & 158 & 112 \\
\hline $\begin{array}{l}\text { Total fruits, berries and total } \\
\text { vegetables }\end{array}$ & 370 & 184 & 369 & 200 \\
\hline $\begin{array}{l}\text { Total fruits and berries, total } \\
\text { vegetables and juices }\end{array}$ & 444 & 234 & 430 & 246 \\
\hline Boiled potatoes & 76 & 58 & 76 & 61 \\
\hline Fried and deep fried potatoes & 0 & 20 & 0 & 20 \\
\hline High-fibre bread & 34 & 43 & 34 & 39 \\
\hline Low-fibre bread & 41 & 50 & 40 & 49 \\
\hline Total bread & 84 & 55 & 82 & 56 \\
\hline High-fibre cereals & 37 & 42 & 37 & 42 \\
\hline Low-fibre cereals & 60 & 56 & 61 & 52 \\
\hline Buns and cookies & 32 & 39 & 32 & 36 \\
\hline Rice/pasta & 7 & 15 & 8 & 15 \\
\hline Nuts & 0 & 1.2 & 0 & 1.1 \\
\hline Snacks & 0 & 0.1 & 0 & 0.5 \\
\hline Fibre & 18.2 & 7.8 & 18.4 & 7.8 \\
\hline
\end{tabular}

the quintiles of the total fruit and vegetables variable was significantly associated with breast cancer risk (Table 2).

Excluding women less than 51 years at baseline, women diagnosed with incident breast cancer within 1 year from baseline examination or cases with in situ breast cancer did not change risk estimates for high fibre intakes. However, the trend across fibre quintiles became significant when we excluded in situ cases or women below 51 years of age at baseline.

Table 3 shows the breast cancer risk in each cell of crossclassified tertiles of fibre and fat intakes. Compared with the group 'high fibre-low fat', all other combinations had elevated estimates. Adjusting for quintiles of energy-adjusted n-6 PUFAs and fermented milk or wine intake categories only marginally influenced outcome, and the risk estimates became slightly lower (data not shown). The test for interaction between tertiles of fibre and fat was significant, $P=0.049$.

\section{DISCUSSION}

We found that high fibre intakes, quintile five compared with quintile one, was associated with a significant $40 \%$ reduction in breast cancer risk. The median fibre intake in quintile five was $25.9 \mathrm{~g} \mathrm{day}^{-1}$, which corresponds to the lower limit of the recommended daily fibre intake in the Nordic countries (Nordiska ministerrådet, 1996). The lower risk associated with high fibre intakes was independent of total fat, n-6 PUFAs, fermented milk and wine intakes. When we examined the combined effect of fibre and fat by cross-classifying tertiles of fibre and fat, the combination high fibre-low fat had the lowest risk of breast cancer. There were no significant associations between intakes of any of the plant food subgroups and breast cancer risk. Ad hoc analyses showed that the protective effect of high fibre intakes remained when we adjusted for intakes of antioxidants (ascorbic acid, $\beta$-carotene, selenium, $\alpha$-tocopherol and folate; data not shown).

The results from epidemiological studies are compatible both with a protective effect, and with no effect, of fibre on breast cancer risk. For instance, in a case-control study in China, women in the lowest tertile of crude fibre intake and highest tertile of fat intake 
Plant foods, fibre and fat and risk of breast cancer

Table 2 Incidence rate ratios of postmenopausal breast cancer according to energy-adjusted quintiles of intakes of fibre and 'total fruit and vegetables' in the Malmö Diet and Cancer cohort 199|-200।

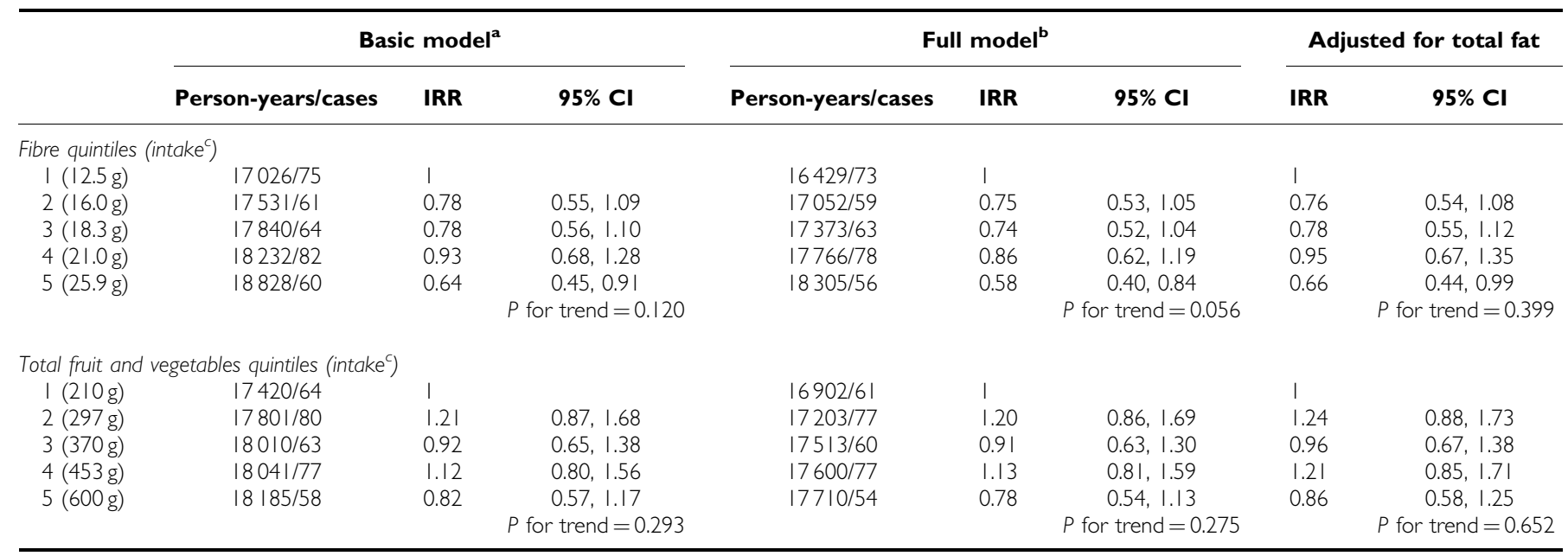

${ }^{a}$ Adjusted for diet interviewer, season of diet interview, method version, age, change of dietary habits. ${ }^{b}$ Adjusted for diet interviewer, season of diet interview, method version, age, change of dietary habits, total energy, current hormone use, age at first child, height, waist, leisure time physical activity, age at menarche, educational level. ' $\mathrm{C}$ rude median intakes of fibre or fruit and vegetables.

Table 3 Incidence rate ratios ${ }^{\mathrm{a}}$ of postmenopausal breast cancer according to combined fibre and fat intake tertiles in the Malmö Diet and Cancer cohort $|99|-200 \mid$

\begin{tabular}{|c|c|c|c|c|c|c|c|c|c|}
\hline \multirow{2}{*}{ Fibre tertiles (fiber intake ${ }^{c}$ ) } & \multicolumn{9}{|c|}{ Fat tertiles $\left(E \%^{b}\right)$} \\
\hline & \multicolumn{3}{|c|}{ I (32.0) } & \multicolumn{3}{|c|}{$2(37.5)$} & \multicolumn{3}{|c|}{$3(42.9)$} \\
\hline I (I3.7) & 1.50 & $0.82,2.73$ & $3868 / 14$ & 1.01 & $0.60,1.71$ & $8058 / 20$ & 1.88 & $1.29,2.73$ & $15793 / 72$ \\
\hline $2(18.3)$ & 1.26 & $0.79,2.02$ & $8117 / 27$ & 1.78 & $1.21,2.63$ & $1|84| / 56$ & 1.35 & $0.86,2.11$ & $9061 / 32$ \\
\hline $3(23.8)$ & 1 & & $17561 / 50$ & 1.56 & $1.02,2.37$ & $9090 / 39$ & 1.99 & $1.17,3.38$ & $3538 / 19$ \\
\hline
\end{tabular}

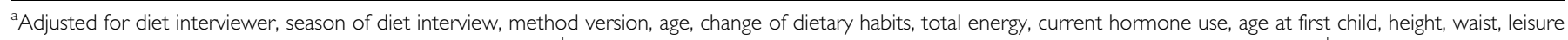
time physical activity, age at menarche and educational level. ${ }^{b}$ Crude median energy percentage of fat (\%). ${ }^{c} \mathrm{Crude}$ median intakes of fibre (g day ${ }^{-1}$ ).

had a 2.9-fold increased risk for breast cancer relative to those in the highest tertile of crude fibre intake and lowest tertile of fat intake (Yuan et al, 1995). Howe et al (1990) found a significant $(P=0.002)$ protective effect of fibre in a combined analysis of 12 case-control studies, but this effect was not independent of $\beta$ carotene and vitamin C. No association was found in the Nurse's Health Study (Willett et al, 1992). A Canadian prospective study found a significant reduced risk in the highest fibre quintile relative to the lowest quintile (Rohan et al, 1993).

There are several hypotheses on the influence of fibre on breast cancer risk. Fibre might modulate the enterohepatic recirculation of oestrogens leading to increased faecal excretion of oestrogens and reduced levels of circulating bioavailable oestrogen (Gerber, 1998). Breast cancer has been suggested to be associated with the insulin resistance syndrome (Kaaks, 1996; Stoll, 1999); a fibre-rich diet could slow digestion and absorption of carbohydrates and influence plasma insulin response (Slavin, 2000). Minor constituents, like antioxidants, phytate and especially phyto-oestrogens, present in fibre-rich foods, might be biologically active components (Gerber, 1998; Cohen, 1999). Phyto-oestrogens (plant food components like isoflavones, lignans and stilbenes) might act both as partial oestrogen agonists and as antagonists, which makes it difficult to predict their effect (Cassidy et al, 2000). Lignans are present in most fibre-rich foods and they might be important sources of phyto-oestrogens, especially in Northern European diets. Epidemiological studies on phyto-oestrogens and breast cancer are scarce and prospective studies have until now shown nonsignificant results (Peeters et al, 2003).

We cross-classified tertiles of fibre and fat in order to illustrate the influence of different fibre and fat intake combinations on breast cancer risk. Risk estimates in groups with low fibre and high fat intakes were lower than expected as indicated by the significant test for interaction between fibre and fat tertiles (i.e., not a multiplicative effect). Although the combination high fibre-low fat had the lowest risk, there is no obvious pattern in breast cancer risk across the nine categories, which implies that other factors also might influence the observed risk estimates. There was no large difference in $n-6 / n-3$ ratio across the nine categories. It varied from 4.99 in the medium fibre-high fat group to 5.25 in the medium fibre-low fat group. However, wine intakes varied substantially across the nine groups. There was a tendency of lower prevalence of high wine intakes with both increasing fibre intakes and increasing fat intakes. The prevalence of high wine intakes was $7.2 \%$ in the low fibre-low fat group and $0.0 \%$ in the high fibre-high fat group. Fermented milk intakes tended to decrease with higher fat intakes and increase with higher fibre intakes. The highest mean intake was found in the medium fibrelow fat group and the lowest mean intake in the medium fibrehigh fat group. This is an interesting finding since it is possible that fermented milk influences the gut flora, which could lead to an increased activation of dietary lignans to active mammalian lignans (Cassidy et al, 2000; Parr and Bolwell, 2000; Adlercreutz, 
126

2002). In fact, a case-control study found a synergistic protective effect of fibre and fermented milk on breast cancer risk. The study concluded that a dietary pattern combining low fat, high fibre and fermented milk might provide substantial protection against breast cancer (van't Veer et al, 1991). Although the extended model was adjusted for these nutrients, residual confounding might remain. It is also plausible that intakes of other nutrients and foods vary across the nine groups and contributes to the risk estimates observed. In addition, the numbers of person-years and cases within each of the nine categories influences the stability of risk estimates. The most stable estimates are found in the high fibrelow fat, medium fibre-medium fat and low fibre-high fat groups.

In this study, we found no association between total fruit and vegetable intakes and breast cancer risk. The intakes of fruit and vegetables in the MDC cohort might be too low and too homogenous to examine any effect. As indicated by food intake data from the calibration study within the European Prospective Investigation into Nutrition and Cancer (EPIC), the MDC subcohort has among the lowest intakes of both fruit and vegetables in the EPIC study centres (Agudo et al, 2003).

Although epidemiological data support the apparent inverse association between consumption of fruits and vegetables and breast cancer risk, studies are not consistent overall (World Cancer Research Fund, 1997). For instance, a meta-analysis comprising published data from 26 cohort and case-control studies found a negative association between vegetables and breast cancer risk, but no association between fruit and breast cancer risk (Gandini et al, 2000). A pooled analysis of eight prospective cohort studies found no significant association between vegetable intakes, fruit intakes and risk of breast cancer (Smith-Warner et al, 2001). The amount eaten and the specific choice of fruit and vegetable in the different cohorts might be some of the explanations behind inconsistent results.

During the initial years of recruitment, over $50 \%$ of the participants were recruited by community-directed activities (Manjer et al, 2002). It is plausible that these 'volunteers' were more health conscious and had a more salutatory behaviour compared to those who joined after a personal invitation. The differences seen in the numbers of person-years in the different fibre quintiles might be explained by higher energy-adjusted fibre intakes in women who joined early.

Under-reporting of energy is a major concern when using selfreported dietary data (Black et al, 1991; Black, 2000). In this study, we used energy-adjusted variables, thus trying to reduce the effect of under-reporting. There was no significant difference in EI/BMR ratios across quintiles of fibre or total fruit and vegetables (data not shown). There were small differences across cross-classified fibre and fat tertiles but no obvious pattern; the EI/BMR ratio varied from 1.41 in the high fibre-high fat category to 1.48 in the high fibre-medium fat category. Although an effect never can be excluded, it is not likely that under-reporting may have a major impact on the outcomes of this study.

To conclude, a dietary pattern characterised by high fibre and low fat intakes was associated with a lower risk of postmenopausal breast cancer. The low intake levels of fruit and vegetables in the MDC cohort may contribute to the absence of an association between breast cancer risk and intakes of fruit and vegetables in this study.

\section{REFERENCES}

Adlercreutz H (2002) Phyto-oestrogens and cancer. Lancet 3: 364-373

Agudo A, Slimani N, Ocké MC, Naska A, Miller AB, Kroke A, Bamia C, Karalis P, Vineis P, Palli D, Bueno-de-Mesquita BH, Peeters PHM, Engeset D, Hjartåker A, Navarro C, Martinez Garcia C, Wallström P, Zhang JX, Welch A, Spencer EA, Stripp C, Overvad K, Clavel-Chapelon E, Casagrande C, Riboli E (2003) Consumption of vegetable, fruit and other plant foods in the European Prospective Investigation into Cancer and Nutrition (EPIC) cohorts from 10 European countries. Public Health Nutr 5: $1179-1196$

Black AE (2000) Critical evaluation of energy intake using the Goldberg cut-off for energy intake:basal metabolic rate. A practical guide to its calculation, use and limitations. Int J Obes 24: 1119-1130

Black AE, Goldberg GR, Jebb SA, Livingstone MBE, Cole TJ, Prentice AM (1991) Critical evaluation of energy intake data using fundamental principles of energy physiology: 2. Evaluating the results of published surveys. Eur J Clin Nutr 45: 583 - 599

Cassidy A, Hanley B, Lamuela-Raventos RM (2000) Isoflavones, lignans and stilbenes - origins, metabolism and potential importance to human health. J Sci Food Agric 80: $1044-1062$

Cohen LA (1999) Dietary fiber and breast cancer. Anticancer Res 19: 3685 3688

Elmståhl S, Riboli E, Lindgärde F, Gullberg Bo, Saracci R (1996) The Malmö food study: the relative validity of a modified diet history method and an extensive food frequency questionnaire for measuring food intake. Eur J Clin Nutr 50: $143-151$

FAO/WHO/UNU (1985) Energy and protein requirements. Report of a joint FAO/WHO/UNU expert consultation, Technical Reports Series, WHO, Geneva

Gandini S, Merzenich H, Robertson C, Boyle P (2000) Meta-analysis of studies on breast cancer risk and diet: the role of fruit and vegetable consumption and the intake of associated micronutrients. Eur J Cancer 36: $636-646$

Gerber M (1998) Fibre and breast cancer. Eur J Cancer Prevent 7: S63-S67 Haftenberger M, Schuit AJ, Tormo MJ, Boeing H, Wareham N, Bueno-deMesquita $\mathrm{BH}$, Kumle M, Hjartåker A, Chirlaque MD, Ardanaz E, Andrén C, Lindahl B, Peeters PHM, Allen N, Overvad K, Tjonneland A, ClavelChapelon E, Linseisen J, Bergmann MM, Trichopoulou A, Lagiou A,
Salvini S, Panico S, Riboli E, Ferrari P, Slimani N (2002) Physical activity of subjects aged 50-64 years involved in the European Prospective Investigation into Cancer and Nutrition (EPIC). Public Health Nutr 5: $1163-1177$

Howe GR, Hirohata T, Hislop GT, Iscovich JM, Yuan J-M, Katsouyanni K, Lubin F, Marubini E, Modan B, Rohan TE, Toniolo P (1990) Dietary factors and risk of breast cancer: combined analysis of 12 case-control studies. J Natl Cancer Inst 82: 561 - 569

Kaaks R (1996) Nutrition, hormones, and breast cancer: is insulin the missing link? Cancer Causes Control 7: 605-625

Lee MM, Lin SS (2000) Dietary fat and breast cancer. Annu Rev Nutr 20: $221-248$

Manjer J, Elmståhl S, Janzon L, Berglund G (2002) Invitation to a population-based cohort study: differences between subjects recruited using various strategies. Scand J Public Health 30: 103-112

Mattisson I, Wirfält E, Andrén C, Gullberg Bo, Berglund G (2003a) Dietary fat intake - food sources and dietary correlates in the Malmö Diet and Cancer cohort. Public Health Nutr 6: 559-569

Mattisson I, Wirfält E, Gullberg Bo, Berglund G (2001) Fat intake is more strongly associated with life style factors than with socio-economic characteristics, regardless of energy adjustment approach. Eur J Clin Nutr 55: $452-461$

Mattisson I, Wirfält E, Wallström P, Gullberg B, Olsson H, Berglund G (2003b) High fat and alcohol intakes are both risk factors of breast cancer in post-menopausal women - a prospective study in the Malmö Diet and Cancer Cohort.

Merlo J, Berglund G, Wirfält E, Gullberg Bo, Hedblad Bo, Manjer J, Hovelius B, Janzon L, Hanson BS, Östergren P-O (2000) Selfadministered questionnaire compared with a personal diary for assessment of current use of hormone therapy: an analysis of 16,060 women. Am J Epidemiol 152: 788-792

Morabia A, Flandre P (1992) Misclassification bias related to definition of menopausal status in case-control studies of breast cancer. Int $J$ Epidemiol 21: $222-228$

Nordiska ministerrådet (Nordic Council of Ministers) (1996) Nordiska näringsrekommendationer 1996 (Nutrient recommendations for the Nordic countries). 28, Nord, Copenhagen (in Swedish) 
Parr AJ, Bolwell PG (2000) Phenols in the plant and in man. The potential for possible nutritional enhancement of the diet by modifying the phenols content or profile. J Sci Food Agric 80: 985-1012

Peeters PHM, Keinan-Boker L, van der Schouw YT, Grobbee DE (2003) Phytoestrogens and breast cancer. Breast Cancer Res Treat 77: $171-183$

Riboli E, Elmståhl S, Saracci R, Gullberg Bo, Lindgärde F (1997) The Malmö food study: validity of two dietary assessment methods for measuring nutrient intake. Int J Epidemiol 26: S161 - S173

Richardsson MT, Leon AS, Jacobs Jr DR, Ainsworth BE, Serfass R (1994) Comprehensive evaluation of the Minnesota Leisure Time Physical Activity Questionnaire. I Clin Epidemiol 47: 271-281

Rohan TE, Howe GR, Friedenreich CM, Jain MG, Miller AB (1993) Dietary fiber, vitamins $\mathrm{A}, \mathrm{C}$ and $\mathrm{E}$, and risk of breast cancer: a cohort study. Cancer Causes Control 4: 29-37

Slavin JL (2000) Mechanisms for the impact of whole grain foods on cancer risk. J Am College Nutr 19: 300S-307S

Smith-Warner SA, Spiegelman D, Shiaw-Shyuan Y, Adami H-O, Beeson LW, van den Brandt PA, Folsom AR, Fraser GE, Freudenheim JL, Goldbohm AR, Graham S, Miller AB, Potter JD, Rohan TE, Speizer FE, Toniolo P, Willett WC, Wolk A, Zeleniuch-Jacquotte A, Hunter DJ (2001) Intake of fruits and vegetables and risk of breast cancer. A pooled analysis of cohort studies. JAMA 285: 769-776

Stoll BA (1996) Obesity and breast cancer. Int J Obes Relat Metab Disord 20: $389-392$

Stoll BA (1999) Western nutrition and the insulin resistance syndrome: a link to breast cancer. Eur J Clin Nutr 53: 83-87
Taylor HL, Jacobs Jr DR, Schucker B, Knudsen J, Leon AS, Debacker G (1978) A questionnaire for the assessment of leisure time physical activities. J Chronic Dis 31: $741-755$

van't Veer P, van Leer EM, Rietdijk A, Kok FJ, Schouten EG, Hermus RJJ, Sturmans F (1991) Combination of dietary factors in relation to breastcancer occurrence. Int J Cancer 47: 649-653

Willett WC (2001) Diet and breast cancer. J Intern Med 249: 395-411

Willett WC, Hunter DJ, Stampfer MJ, Colditz GA, Manson JE, Spiegelman D, Rosner BA, Hennekens CH, Speizer FE (1992) Dietary fat and fiber in relation to risk of breast cancer. J Am Med Assoc 268: 2037 - 2044

Wirfält E, Mattisson I, Gullberg Bo, Johansson U, Olsson H, Berglund G (2002a) Post-menopausal breast cancer is associated with high intakes of $\omega-6$ fatty acids. Cancer Causes Control 13: $883-893$

Wirfält E, Mattisson I, Gullberg B, Olsson H, Berglund G (2003) Food sources of fat and postmenopausal breast cancer. Conference Proceeding of the 5th International Conference on Dietary Assessment Methods, Chiang Rai, Thailand

Wirfält E, Mattisson I, Johansson U, Gullberg Bo, Wallström P, Berglund G (2002b) A methodological report from the Malmö Diet and Cancer study: development and evaluation of altered routines in dietary data processing. Nutr J 1: 3. www.nutritionj.com

World Cancer Research Fund (1997) Breast. In Food, Nutrition and the Prevention of Cancer: A Global Perspective, Potter JD (ed) pp 252-287. Washington: World Cancer Research Fund/American Institute for Cancer Research

Yuan J-M, Wang QS, Ross RK, Henderson BE, Yu MC (1995) Diet and breast cancer in Shanghai and Tianjin, China. Br J Cancer 71: 1353-1358 